\title{
On the relations between the numbers of standard tableaux
}

\author{
By D. E. RUTherford.
}

(Received 4th August, 1941. Read 1st November, 1941.)

$\S 1$. Corresponding to any partition

$$
a_{1}+a_{2}+\ldots+\alpha_{k}=n, \quad a_{1} \geqq a_{2} \geqq \ldots \geqq a_{k},
$$

which we denote by $\left[a_{1}, \ldots, a_{k}\right]$ or briefly by $[\alpha]$, of the integer $n$, we can construct a shape which has $a_{1}$ spaces in the first row, $a_{2}$ in the second row, $\ldots, a_{k}$ in the $k^{\text {th }}$ and last row. Thus the shape corresponding to the partition $[5,3,3,2]$ of 13 has the form:

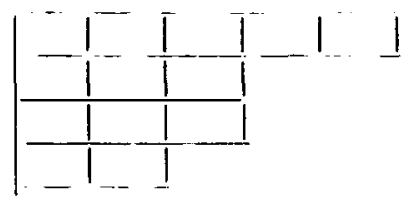

We can obtain $n$ ! tableaux from a given shape [a] by arranging the letters $1,2, \ldots, n$ in the $n$ spaces in all possible ways. Of these $n$ ! tableaux there is a certain number $f_{[a]}$ of them each of which has the property that the letters in each row and in each column are in numerical order. Such tableaux are said to be standard. Thus

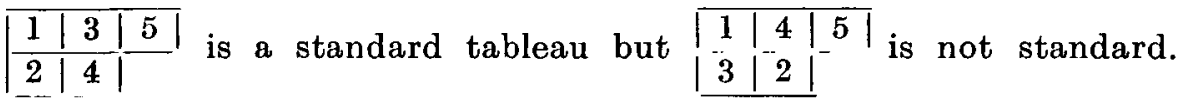

This number $f_{[\alpha]}$ plays an important rôle in the theory of the representations of the symmetric group of order $n$ ! for it turns out that $f_{[a]}$ is the order of the irreducible representation associated with the partition $[a]$. The above concept of a tableau was originated and developed by the late Alfred Young in a series of very interesting papers $^{1}$ dealing with the irreducible representations of the symmetric group.

The purpose of this paper is to obtain the three formulae (A), (B), (C) by new methods. The proof of (A) is a modification of that used by Young, worked out by Dr I. M. H. Etherington and Dr W. Ledermann while we were on holiday together. So far as I am aware,

1 Proc. London Math. Soc., 33 (1901), 97 ; 34 (1902), 361 ; (2) 28 (1928), 255 ; (2) 31 (1930), 253 ; (2) 34 (1932), 196 ; (2) 37 (1934), 441 ; and other papers. 
the proofs here given of (B) and (C) are new. They are interesting on account of their simplicity. The proof in each case is by induction and the basis of the inductions is the trivial result:

$$
f_{[1]}=f_{[2]}=f_{[1,1]}=1 \text {. }
$$

§2. We observe that in a standard tableau the last letter $n$ must appear at the extreme right of the row in which it occurs and at the foot of the column in which it occurs. By removing this letter we automatically obtain a standard tableau of $n-1$ letters, for the remaining letters are still in numerical order in each row and in each column. Thus, following Young, we obtain the relationship

$$
f_{[a]}=\sum_{\lambda=1}^{k} f_{\left[a_{\lambda}-\right]},
$$

where $\left[a_{\lambda}-\right]$ denotes the partition $\left[a_{1}, \ldots, a_{\lambda}-1, \ldots, a_{k}\right]$ of $n-1$. A word of explanation is necessary here, for if $a_{\lambda}=a_{\lambda+1}$, then $a_{\lambda}-1<a_{\lambda+1}$, and we do not recognise tableaux in which any row is shorter than the one following it. In such cases we define $f_{\left[a_{\lambda}-\right]} \equiv 0$, in order that we may sum from 1 to $k$ in (2).

We shall now prove by induction the relation

$$
f_{[a]}=n ! \frac{\prod_{r<s \leqq k}\left(x_{r}-x_{s}\right)}{\prod_{r} x_{r} !},
$$

where we define

$$
x_{r} \equiv a_{r}+k-r .
$$

It will be observed that in the case where $a_{\lambda}=a_{\lambda+1}$ the formula (A) yields $f_{\left[a_{\lambda}-\right]}=0$ on account of a vanishing factor $\left(x_{\lambda}-x_{\lambda+1}\right)$. This is in agreement with our previous definition. We may mention here also that if we write $\left[a_{1}, \ldots, a_{k}\right]$ in the form $\left[a_{1}, \ldots, a_{k}, a_{k+1}\right]$ with $a_{k+1}$ equal to zero, the right hand side of (A) retains the same numerical value.

Assuming the truth of the relation (A) in the case of $n-1$ letters, we can readily show that

$$
f_{\left[\alpha_{\lambda}-\right]}=\theta \frac{x_{\lambda}}{n} \prod_{s \neq \lambda} \frac{\left(x_{\lambda}-1-x_{g}\right)}{\left(x_{\lambda}-x_{s}\right)},
$$

where $\theta$ denotes the right hand side of (A). We have to show that $\theta$ is in fact the number $f_{[a]}$ of standard tableaux associated with the partition [a] of $n$. From (2) we deduce that 


$$
f_{[a]}=\frac{\theta}{n} \sum_{\lambda} x_{\lambda} \prod_{\delta \neq \lambda} \frac{\left(x_{\lambda}-1-x_{s}\right)}{\left(x_{\lambda}-x_{s}\right)}=-\frac{\theta}{n} \sum_{\lambda} \frac{x_{\lambda} \phi\left(x_{\lambda}-1\right)}{\phi^{\prime}\left(x_{\lambda}\right)},
$$

where we write

$$
\phi(x) \equiv\left(x-x_{1}\right) \ldots\left(x-x_{k}\right) .
$$

Now since $x^{2} \phi(x-1)$ is of degree $k+2$ in $x$, we can write

$$
\frac{x^{2} \phi(x-1)}{\phi(x)}=Q(x)+\frac{R(x)}{\phi(x)}
$$

where the quotient $Q(x)$ is of degree 2 and the remainder $R(x)$ is of degree $k-1$ or less. On multiplying throughout by $\phi(x)$ and then putting $x=x_{\lambda}$, we have immediately

$$
R\left(x_{\lambda}\right)=x_{\lambda}^{2} \phi\left(x_{\lambda}-1\right) .
$$

Since the zeros $x_{1}, \ldots, x_{k}$ of $\phi(x)$ are distinct, it follows from the theory of partial fractions that

$$
\frac{R(x)}{\phi(x)}=\sum_{\lambda=1}^{k} \frac{R\left(x_{\lambda}\right)}{\left(x-x_{\lambda}\right) \phi^{\prime}\left(x_{\lambda}\right)}=\sum_{\lambda=1}^{k} \frac{x_{\lambda}^{2} \phi\left(x_{\lambda}-1\right)}{\left(x-x_{\lambda}\right) \phi^{\prime}\left(x_{\lambda}\right)} .
$$

Substituting this value in (4) and putting $x=0$, we have

$$
\sum_{\lambda} \frac{x_{\lambda} \phi\left(x_{\lambda}-1\right)}{\phi^{\prime}\left(x_{\lambda}\right)}=Q(0) \text {. }
$$

Now for suitable values of $x$,

$$
\begin{aligned}
\frac{\phi(x-1)}{\phi(x)} & =\prod_{\lambda}\left(1-\frac{1}{x-x_{\lambda}}\right) \\
& =\prod_{\lambda}\left(1-\frac{1}{x}-\frac{x_{\lambda}}{x^{2}}-\ldots\right),
\end{aligned}
$$

and $Q(0)$ is the coefficient of $1 / x^{2}$ in this product. Thus

since

$$
\begin{gathered}
Q(0)=\frac{1}{2} k(k-1)-\sum_{\lambda} x_{\lambda}=-n, \\
\sum_{\lambda} x_{\lambda}=\sum_{\lambda}\left(a_{\lambda}+k-\lambda\right)=n+\frac{1}{2} k(k-1) .
\end{gathered}
$$

Combining equations (3), (5) and (6), we obtain the desired relation

$$
f_{[a]}=\theta \text {. }
$$

$\S 3$. We now proceed to prove the formula

$$
(n+1) f_{[a}=\sum_{\lambda=1}^{k+1} f_{\left[a_{\lambda}+\right]}
$$

where $\left[a_{\lambda}+\right]$ denotes the partition $\left[a_{1}, \ldots, a_{\lambda}+1, \ldots, a_{k+1}\right]$ of 
$n+1$ in which $a_{k+1}$ vanishes. Assuming the truth of the theorem in the case of $n-1$. letters, we have from (2)

$$
f_{\left[a_{\lambda}+\right]}=\Sigma \int_{\left[a_{\lambda}+, a_{\mu}-\right]}
$$

where $\left[a_{\lambda}+, a_{\mu}-\right]$ denotes $\left[a_{1}, \ldots, a_{\lambda}+1, \ldots, a_{\mu}-1, \ldots, a_{k+1}\right]$ if $\lambda \neq \mu$ and $\left[a_{\lambda}+, a_{\lambda}-\right]$ is simply [a]. In the last formula $\mu$ is summed from 1 to $k$ except when $\lambda=k+1$, in which case $\mu$ is summed from 1 to $k+1$. Thus

$$
\sum_{\lambda=1}^{k+1} f_{\left[a_{\lambda}+\right]}=\sum_{\lambda=1}^{k+1} \sum_{\mu=1}^{k} f_{\left[a_{\lambda}+, a_{\mu}-\right]}+f_{[a]} .
$$

But by hypothesis

$$
\begin{gathered}
\sum_{\lambda=1}^{k+1} f_{\left[a_{\lambda}+, a_{\mu}-\right]}=n f_{\left[\alpha_{\mu}-\right]}, \\
\sum_{\lambda=1}^{k+1} f_{\left[a_{\lambda}+\right]}=n \sum_{\mu=1}^{k} f_{\left[a_{\mu}-\right]}+f_{[a]}=(n+1) f_{[a]},
\end{gathered}
$$

so,

which establishes the induction.

$\S 4$. We now deduce the formula

$$
\sum_{[a]} f_{[a]}^{2}=n !
$$

the summation being taken over all partitions [a] of $n$. We assume the theorem true for $n$ and obtain from (B) the relation

$$
\sum_{[a]} \sum_{\lambda=: 1}^{k+1} f_{\left[a_{\lambda}+\right]} f_{[a]}=(n+1) \sum_{[a]} f_{[a]}^{2}=(n+1) \text { !, }
$$

where, it must be remembered, $k$ is number of rows in the shape [a]. Now by a rearrangement of terms, the double summation may also be expressed in the form

$$
\sum_{[\beta]} \sum_{\mu=1}^{l} f_{\left[\beta_{\mu}-\right]} f_{[\beta]}
$$

where $[\beta]$ denotes a partition $\left[\beta_{1}, \ldots, \beta_{l}\right]$ of $n+1$. (For if $\left[\alpha_{\lambda}+\right]=[\beta]$, then $\left[\beta_{\lambda}-\right]=[\alpha]$, so that each term in the first double summation occurs once and once only in the second double sum mation, and vice versa).

From (2), we have

$$
\sum_{[\beta]} \sum_{\mu=1}^{l} f_{\left[\beta_{\mu}-\right]} f_{[\beta]}=\sum_{[\beta]} f_{[\beta]}^{2}=(n+1) ! ;
$$

and this concludes the induction.

The UNIVERsity,

ST ANDREWS. 